\title{
Article
}

\section{Gallery of integrating factors for non-linear first-order differential equations}

\author{
Albert Adu-Sackey ${ }^{1}$, Gabriel Obed Fosu ${ }^{2, *}$ and Buckman Akuffo ${ }^{1}$ \\ 1 Department of Applied Mathematics, Koforidua Technical University, Ghana. \\ 2 Department of Mathematics, Kwame Nkrumah University of Science and Technology, Ghana. \\ * Correspondence: gabriel.obed@presbyuniversity.edu.gh
}

Received: 22 March 2021; Accepted: 27 November 2021; Published: 30 December 2021.

\begin{abstract}
This paper discusses a gallery of useful results in connection with integrating factors that are often left as problems for discovery learning and are generally not taught in typical Ordinary Differential Equations courses. Most often than not the approach earlier writers employ is to give a possible form for an integrating factor that may results in an integrating curve without practical prove as far as the subject matter is concerned. In this write-up, an attempt is made by solving the resulting partial differential equation emanating from an underlining general differential equation of a non-exact form, by the use of the ratio theorem to establish various intricate possibilities of integrating factors that are seldom and often relegated to the background, even though they may be equally be applied as a function of a unitary variable or a linear combination of both the dependent and independent variables under certain conditions. Granted an integrating factor is found and such a function applied, the benefit is enormous especially the non-exact differential equation reduces into a known type which may be identified as exact, homogeneous, and or separable that yields a solution.
\end{abstract}

Keywords: Integrating factor, Ratio theorem, ODE, PDE.

\section{Introduction and preliminaries}

$\mathbf{T}$ he extreme challenge in finding an integrating factor of a simple or complex form, for a non-exact differential equation is borne out of solving a partial differential equation base on certain conditionality imposed on the ordinary differential equation (ODE) to transform it into an exact type. The snappy introduction of partial differential equation in the study of ordinary differential equations may pose some difficulty [1] of a sort to people who may only be taking the course briefly and may not advance or stray into the study of calculus of several variables, mathematical methods, integral equations, and specialized fields like special functions of mathematical physics, thermodynamics, and quantum mechanics. These advanced areas require a grave deal of useful repertoire of the methods or the techniques that provides a complete procedure for solving mercenaries of differential equations [2-4]. For instance, the functions that are usually classified as special functions of mathematical physics are all characterized by second-order differential equations and are implicit in the use of transforming their forms into exactness, leading to the derivation of their orthogonal properties by the application of certain integrating factor.

The use of an integrating factor has the advantage that is susceptible in the handling of most ordinary differential equations. In literature and for practical reasons, simple integrating factors involving a single variable could routinely be found [5]. This makes it possible for the non-exact differential equation to become solvable [6], so that the limitation or difficulty of having to deal with the partial differential equation in order to extract the possible integrating factor(s) from is completely taking care of. This fact makes it unattractive and a quick avenue for the subject matter to be touched on and short-lived in favor of the other aspects of the study of ordinary differential equations. Such intervention and notion may rather put readers at a disadvantage in general for two main reasons.

The first being that, readers may run along with the idea that once a first order non-linear equation is seen not to be exact nor meet the litmus test of the known types with their tailored appropriate methods that go with them, in the determination of their respective solutions, then one can always obtain an integrating factor 
in a single variable only or for want of a better word, by inspection [5,7], the entire equation is re-arranged into an integral form and the solution deduced without fully giving such method the in-depth attention it fully deserves as is often the case.

Secondly, the passion for a possible extension for the subject matter especially for integrating factors in two or more variables as well as the development of such techniques for higher-order differential equations may be completely incomprehensible or out of reach, even though quiet an extensive work has been done by earlier writers, particularly in the reduction of higher orders of ordinary differential equations under symmetric considerations [8,9]. The interesting aspect of the use of symmetric conditions for systems of ordinary or partial differential equation is that they tell much about the closed-form or analytic solvability of the system without having to routinely solve it in advance $[1,10]$. In contrast, this parallels the approach for the determination of integrating factors, in the sense that if there exist infinitely many integrating factors to the same non-exact differential equation, then its general solution may be obtained in terms of any two of such functions expressed as a direct proportion without actually solving the given differential equation.

An integrating factor is guaranteed to exist provided the given differential equation is solvable and in fact the general conditions of existence of integrating factors are derived under the theory of Lie group and Lie symmetries $[1,11,12]$. Once such a function is determined and applied, the exact equation now obtain, play out as a total derivative [13] of some appropriate function referred to as the potential function [14,15]. The resulting general solution from this procedure is implicitly [5] defined and geometrically it represents a family of level curves for that potential function [6,11].

In this paper, we shall primarily dwell on first order differential equations by resorting to a combination of partial differential equation and the ratio theorem to establish various integrating factors, of great value which could remarkably be applied in reducing a given differential equation into exactness and hence separable form for its solution to be determined

A cursory look at differential calculus reveals that there is no one size fit straight jacket approach or in general an algorithm in the determination of integrating factors [16] for nonlinear first-order differential equations. Hither, we shall attempt to construct a variety of non-zero [6] functions from the necessary and sufficient conditions that require a none exact differential equation to convert into an exact form. We shall extensively apply the theory on ratios and more importantly to identify certain exact forms to develop a number of cases involving the use of integrating factor formulas, some of which are obviously going to be in terms of a single variable whiles others may appear as sums and products or quotients involving linear combinations of two variables of the types $x y, x / y, y \pm x$, and $y^{2} \pm x^{2}$.

\section{Forms of integrating factors}

Let the differential form of a first-order differential equation assumed to be non-exact be given by

$$
M(x, y) d x+N(x, y) d y=0 .
$$

Then the necessary and sufficient condition for Equation (1) to transform into exact is based on the partial differential relation

$$
[\mu M(x, y)]_{y}=[\mu N(x, y)]_{x}
$$

where $\mu(x, y)$ is an integrating factor, and $M, N$ are arbitrary functions with continuous first derivatives within a closed simply connected region in $x$ and $y$. Once this condition criterion is satisfied, Equation (2) expands into

$$
M \mu_{y}-N \mu_{x}=\mu\left[N_{x}-M_{y}\right] .
$$

The auxiliary equation associated with the resulting expansion in (3) yields

$$
\frac{d x}{N}=\frac{d y}{-M}=\frac{d \mu}{\mu\left[M_{y}-N_{x}\right]}
$$

Equation (4) may be hinged upon as a basis to afford us the opportunity to develop a number of useful cases of integrating factor types that can be deduced to make the non-exact differential equation (ODE) transforms 
into exact form depending on the nature of the referenced differential equation as given by Equation (1). The cases are expounded below:

\section{Case 1}

If we seek an integrating factor purely in $x$, then by pairing the first and last expressions in Equation (4) we get

$$
\frac{d x}{N}=\frac{d \mu}{\mu\left[M_{y}-N_{x}\right]} \Longrightarrow \mu(x)=e^{\int f(x) d x},
$$

where $f(x)=\left(M_{y}-N_{x}\right) / N$. This integrating factor (5) would always transform the standard first-order non-exact differential equation into an exact type, provided it is expressed in the same appearance and form of Equation (1), that is $[p(x) y-q(x)] d y+d x=0[4,17]$. The result of the transformed equation has the benefit of always yielding a unique solution without any singularity [13] defined in them whenever, the coefficients $p(x)$ and $q(x)$ are continuous on a prescribed open interval I [4,17].

\section{Case 2}

For an integrating factor in the dependent variable $y$ alone, one may similarly take from Equation (4) the relation

$$
\frac{d y}{-M}=\frac{d \mu}{\mu\left[M_{y}-N_{x}\right]} \Longrightarrow \mu(x)=e^{\int f(y) d y}
$$

where $f(y)=\left(\frac{N_{x}-M_{y}}{M}\right)$.

\section{Case 3}

Other combinations are possible by applying the ratio theorem and choosing constant multipliers of 1 for the first two terms. By such multipliers, the Equation (4) is modified into the form

$$
\frac{d x}{N}=\frac{d y}{-M}=\frac{d \mu}{\mu\left[M_{y}-N_{x}\right]}=\frac{d(x \pm y)}{N \pm M} .
$$

Now for integrating factor formulas to be churned out in terms of sums and differences, we shall need to pair the third and fourth expressions in Equation (7) to give

$$
\frac{d \mu}{\mu\left[M_{y}-N_{x}\right]}=\frac{d(x \pm y)}{N \pm M} \Longrightarrow \mu(x \pm y)=e^{\int f(x \pm y) d(x \pm y)},
$$

where $f(x \pm y)=\left(\frac{M_{y}-N_{x}}{N \pm M}\right)$.

\section{Case 4}

For an integrating factor of the combination $x y$, it is necessary to take up the multipliers $y$ and $x$ for the first two terms of Equation (4), and again by pairing the results with the last term of the same equation, one is led to the relation

$$
\frac{d \mu}{\mu\left[M_{y}-N_{x}\right]}=\frac{y d x+x d y}{y N-x M} \Longrightarrow \mu(x y)=e^{\int f(x y) d(x y)},
$$

where $f(x y)=\left(\frac{M_{y}-N_{x}}{y N-x M}\right)$.

\section{Case 5}

For an integrating factor involving sums of squares of the form $x^{2}+y^{2}$, it is necessary to take up the multipliers $2 x$ and $2 y$ for the first two terms of Equation (4), and thus by pairing the results with the last term of the same equation, then it leads to the relation 


$$
\frac{d \mu}{\mu\left[M_{y}-N_{x}\right]}=\frac{2 x d y+2 y d x}{2[x N-y M]} \Longrightarrow \mu\left(x^{2}+y^{2}\right)=e^{\int f\left(x^{2}+y^{2}\right) d\left(x^{2}+y^{2}\right)},
$$

where $f\left(x^{2}+y^{2}\right)=\frac{1}{2}\left(\frac{M y-N_{x}}{x N-y M}\right)$.

\section{Case 6}

It can be seen that the differential equation of an innocently looking form $y d x-x d y=0$ is indeed not exact, yet separable. This equation can be shown to transform into exactness by the use of the following non-trivial [6] integrating factors

$$
\frac{1}{x^{2}}, \frac{1}{y^{2}}, \frac{1}{x^{2}-y^{2}}, \frac{1}{x^{2}+y^{2}} .
$$

These choices of integrating factors for the differential pair $y d x-x d y[7,18,19]$, could be exploited to our advantage in introducing five extra useful integrating formulas defined exponentially. A striking coincidence of such a differential pair is arrived at, by noting that when multipliers $y$ and $-x$ are imposed on the first two terms of the Equation (4) and the resulting expression paired with the last term of that same equation, one is led to the relation

$$
\frac{d \mu}{\mu\left[M_{y}-N_{x}\right]}=\frac{y d x-x d y}{[y N+x M]} \Longrightarrow \mu(\theta)=e^{\int f(\theta) d \theta},
$$

where $f(\theta)=\tau\left(\frac{M y-N_{x}}{y N+x M}\right)$.

Now, when $\tau$ takes on the values $x^{2}, y^{2}, x y, x^{2}-y^{2}, x^{2}+y^{2}$, we obtain five different integrating factors which may be used to convert the non-exact differential Equation (1) into an exact form, where such functions are well-defined as shown below:

1. $\mu\left(\frac{x}{y}\right)=e^{\int f(x / y) d(x / y)}$, where $f\left(\frac{x}{y}\right)=y^{2}\left(\frac{M_{y}-N_{x}}{y N+x M}\right)$;

2. $\mu\left(\frac{y}{x}\right)=e^{\int f(y / x) d(y / x)}$, where $f\left(\frac{y}{x}\right)=x^{2}\left(\frac{M_{y}-N_{x}}{y N+x M}\right)$;

3. $\mu\left(\ln \frac{x}{y}\right)=e^{\int f(\ln (x / y)) d(\ln (x / y))}$, where $f\left(\ln \frac{x}{y}\right)=x y\left(\frac{M_{y}-N_{x}}{y N+x M}\right)$;

4. $\mu\left(\arctan \frac{x}{y}\right)=e^{\int f(\arctan (x / y)) d(\arctan (x / y))}$, where $f\left(\arctan \frac{x}{y}\right)=\left(x^{2}+y^{2}\right)\left(\frac{M_{y}-N_{x}}{y N+x M}\right)$;

5. $\mu(\xi)=e^{\int f(\xi) d \xi}$, where $f(\xi)=\left(y^{2}-x^{2}\right)\left(\frac{M_{y}-N_{x}}{y N+x M}\right)$, and $\xi=\frac{1}{2} \ln \frac{x+y}{y-x}$.

Apart from obtaining these various possibilities for the integrating factor formulas expressed in at least one variable, and in terms of the exponential function in connection with Equation (1), it may be possible to further develop some integrating factors under some characterization associated to the non-exact differential equation. Such cases are considered and proved as follows:

\section{Case 7}

If it is known that the non-exact differential Equation (1) is homogeneous with respect to the coefficients $M(x, y)$, and $N(x, y)$ of the same degree, then the integrating factor takes the form

$$
\mu(x, y)=\frac{1}{x M+y N} .
$$

Proof. For homogeneous functions, we may express $M$ and $N$ as

$$
M(x, y)=x^{n} \phi_{1}(v), N(x, y)=x^{n} \phi_{2}(v), \text { with } v=\frac{y}{x}
$$

Substituting (13) into (1), we obtain

$$
x^{n} \phi_{1}(v) d x+x^{n} \phi_{2}(v)[v d x+x d v]=0 .
$$


This implies that

$$
x^{n}\left[\phi_{1}(v)+v \phi_{2}(v)\right] d x+x^{n+1} \phi_{2}(v) d v=0 .
$$

For Equation (14) to be exact and separable we need to divide through by $x^{n+1}\left[\phi_{1}(v)+v \phi_{2}(v)\right]$, leading to

$$
\frac{1}{x} d x+\frac{\phi_{2}(v) d v}{\left[\phi_{1}(v)+v \phi_{2}(v)\right]}=0
$$

Clearly the form of Equation (15) is separable and so such a divisor must be an integrating factor. That is

$$
\mu(x, y)=\frac{1}{x^{n+1}\left[\phi_{1}(v)+v \phi_{2}(v)\right]}=\frac{1}{x M+y N} .
$$

\section{Case 8}

If it is known that the coefficients of the non-exact differential Equation (1) are functions of products of $x$ and $y$ defined by $M(x, y)=y f_{1}(x y)$, and $N(x, y)=x f_{2}(x y)$, then the integrating factor can readily be shown to be

$$
\mu(x, y)=\frac{1}{x M-y N} .
$$

Proof. By substituting the forms of $M(x, y)$ and $N(x, y)$ together with the expression $\left[y f_{2}(x y)-y f_{2}(x y)\right] d x$, into Equation (1) we get

$$
y f_{1}(x y) d x+\left[y f_{x}(x y)-y f_{2}(x y)\right] d x+x f_{2}(x y) d y=0
$$

Thus

$$
y\left[f_{1}(x y)-f_{2}(x y)\right] d x+f_{2}(x y) d(x y)=0
$$

This implies that

$$
\frac{x y}{x}\left[f_{1}(x y)-f_{2}(x y)\right] d x+f_{2}(x y) d(x y)=0 .
$$

For Equation (17) to be exact and separable, it is expedient to divide through by $x y\left[f_{1}(x y)-f_{2}(x y)\right]$, leading to

$$
\frac{1}{x} d x+\frac{f_{2}(x y)}{x y\left[f_{1}(x y)-f_{2}(x y)\right]} d(x y)=0 .
$$

Clearly the form of Equation (18) is separable and so such a divisor must be an integrating factor. That is

$$
\mu(x, y)=\frac{1}{x y\left[f_{1}(x y)-f_{2}(x y)\right]}=\frac{1}{x M-y N^{\prime}}, \text { provided } x M-y N \neq 0 .
$$

In case the powers of each term of the factors of the product are unequal and have the form $M(x, y)=$ $y f_{1}\left(x^{m} y^{n}\right)$ and $N(x, y)=x f_{2}\left(x^{m} y^{n}\right)$, then the integrating factor can readily be shown to be

$$
\mu(x, y)=\frac{1}{n M_{x}-m N_{y}} .
$$

Proof. By substituting the new forms of $M(x, y)$ and $N(x, y)$ into Equation (1) we get

$$
f_{1}\left(x^{m} y^{n}\right) y d x+f_{2}\left(x^{m} y^{n}\right) x d y=0
$$

Now by writing out the total differential of the product $x^{m} y^{n}$, we see that

$$
d\left(x^{m} y^{n}\right)=m x^{m-1} y^{n} d x+n x^{m} y^{n-1} d y
$$

leads to

$$
x d y=\frac{1}{n x^{m-1} y^{n-1}}\left[d\left(x^{m} y^{n}\right)-m x^{m-1} y^{n-1} \cdot y d x\right]
$$


Using the expression in Equation (20) into Equation (19) and grouping the terms for $d x$ and $d y$ we obtain

$$
\left[f_{1}\left(x^{m} y^{n}\right)-\frac{m}{n} f_{2}\left(x^{m} y^{n}\right)\right] y d x+\frac{1}{n x^{m-1} y^{n-1}} f_{2}\left(x^{m} y^{n}\right) d\left(x^{m} y^{n}\right)=0 .
$$

This is expressed as

$$
\frac{x y}{x}\left[n f_{1}\left(x^{m} y^{n}\right)-m f_{2}\left(x^{m} y^{n}\right)\right] d x+\frac{1}{x^{m-1} y^{n-1}} f_{2}\left(x^{m} y^{n}\right) d\left(x^{m} y^{n}\right)=0 .
$$

This implies that

$$
\frac{1}{x}\left[n f_{1}\left(x^{m} y^{n}\right)-m f_{2}\left(x^{m} y^{n}\right)\right] d x+\frac{1}{x^{m} y^{n}} f_{2}\left(x^{m} y^{n}\right) d\left(x^{m} y^{n}\right)=0 .
$$

Finally, to get separable differentials, we need to divide Equation (21) through by the expression $\left[n f_{1}\left(x^{m} y^{n}\right)-m f_{2}\left(x^{m} y^{n}\right)\right]$. This yields

$$
\frac{1}{x} d x+\frac{f_{2}\left(x^{m} y^{n}\right)}{x^{m} y^{n}\left[n f_{1}\left(x^{m} y^{n}\right)-m f_{2}\left(x^{m} y^{n}\right)\right]} d\left(x^{m} y^{n}\right)=0 .
$$

Thus, the function

$$
\mu(x, y)=\frac{1}{x y\left[n f_{1}\left(x^{m} y^{n}\right)-m f_{2}\left(x^{m} y^{n}\right)\right]}=\frac{1}{n M_{x}-m N_{y}}
$$

must be the integrating factor for Equation (19) provided $n M_{x}-m N_{y} \neq 0$.

\section{Case 9}

An integrating factor of the form $\mu(x, y)=x^{p} y^{q}$ may be assumed if the differential equation is given by the general form

$$
x^{\alpha} y^{\beta}(m y d x+n x d x)+x^{\rho} y^{\sigma}(a y d x+b x d y)=0,
$$

where $\alpha, \beta, \rho, \sigma, m, n, a, b$ are constants, with $p$ and $q$ being unknown constants to be determined. In order to apply such an integrating factor, the strategy [6] is to ensure that the unknown index constants $p$ and $q$ must be selected in such a way that when Equation (23) is multiplied by this integrating factor $\mu(x, y)$ exactness is achieved. That is

$$
\left[m x^{\alpha+p} y^{y^{\beta+q+1}}+a x^{\rho+p} y^{\sigma+q+1}\right]_{y}=\left[m x^{\alpha+p} y^{y^{\beta+q+1}}+a x^{\rho+p} y^{\sigma+q+1}\right]_{x} .
$$

On performing the partial derivatives and equating coefficients of the terms $x^{\alpha+p} y^{\beta+q}$ and $x^{\rho+p} y^{\sigma+q}$, we get the following equations

$$
m q-n p=n(\alpha+1)-m(\beta+1) ; \quad a q-b p=n(\alpha+1)-m(\beta+1) .
$$

Now, provided the determinant of the coefficients of $q$ and $p$ in the systems of linear equations in two unknown is non-singular, that is $\left|\begin{array}{cc}m & n \\ a & b\end{array}\right| \neq 0$, then can $p$ and $q$ be found uniquely from Equation (25) by

$$
\begin{aligned}
& p=\frac{1}{b m-a n}[a m(\sigma-\beta)+a n(\alpha+1)-m b(\rho+1)], \\
& q=\frac{1}{b m-a n}[b n(\alpha-\rho)+a n(\sigma+1)-m b(\beta+1)] .
\end{aligned}
$$

If it happens that the coefficients of the products $x^{m} y^{n}$ and $x^{a} y^{b}$ of the differential pairs of Equation (23) are arbitrarily defined by functions $f_{1}\left(x^{m} y^{n}\right)$ and $f_{2}\left(x^{a} y^{b}\right)$, such that their index indices coincides with the constants associated with the given differential pairs, then it is may be possible to obtain a much simpler integrating factor to the differential equation

$$
f_{1}\left(x^{m} y^{n}\right)(m y d x+n x d y)+f_{2}\left(x^{a} y^{b}\right)(a y d x+b x d y)=0
$$


by observing that the total differentials of $x^{m} y^{n}$ and $x^{a} y^{b}$ can be written as

$$
d\left(x^{m} y^{n}\right)=x^{m-1} y^{n-1}(m y d x+n x d y) ; \quad d\left(x^{a} y^{b}\right)=x^{a-1} y^{b-1}(a y d x+b x d y) .
$$

Now by substituting Equation (27) in (26) and multiplying through by the quantity 1 / $x y$ we obtain

$$
\frac{1}{x^{m} y^{n}} f_{1}\left(x^{m} y^{n}\right) d\left(x^{m} y^{n}\right)+\frac{1}{x^{a} y^{b}} f_{2}\left(x^{a} y^{b}\right) d\left(x^{a} y^{b}\right)=0
$$

Clearly Equation (28) is indeed separable, and so an integrating factor for Equation (26) must be of the form

$$
\mu(x, y)=\frac{1}{x y} .
$$

\section{Case 10}

Some differential forms may not have associated integrating factors to them, but if such differential equations are characterized as having Homogeneous coefficients, then it suffices to fall on certain useful appropriate substitutions, that transform the differential equations into other standard equations which may have readily available integration factors [20] to make them solvable. A consideration of the differential equation of the form

$$
\left[f_{1}(x, y)+f_{3}(x, y) x^{k+1}\right] d y=\left[f_{2}(x, y)+f_{3}(x, y) y x^{k}\right] d x
$$

where $f_{1}(x, y), f_{2}(x, y)$ and $f_{3}(x, y)$ are homogeneous functions of $x$ and $y$, while $f_{1}(x, y)$ and $f_{2}(x, y)$ are of the same degree have no direct integrating factor though, but the use of the common substitution $y=v x$ is priceless when handling homogeneous differential equations. This particular substitution transform Equation (29) into a standard Bernoulli equation which is known to be non-linear and can further be reduced into linear standard equation which of course will still remain non-exact.

Proof. By the hypothesis that the arbitrary functions $f_{1}(x, y), f_{2}(x, y)$ and $f_{3}(x, y)$ are homogeneous, let $y=$ $v x \Longrightarrow d y=v d x+x d v$ and noting from Equation (11) that when Equation (29) is carefully arranged the last term of that equation yields total differential form given by $d(y / x)=(x d y-y d x) / x^{2}=d v$, so that in effect the differential equation for case 10 reduces to the form

$$
\left[f_{1}(v)(v d x+x d v)-f_{2}(v) d x\right]+f_{3}(v) x^{k+2} d v=0
$$

Implying that

$$
\left[v f_{1}(v)-f_{2}(v)\right] d x+x f_{1}(v) d v+f_{3}(v) x^{k+2} d v=0 .
$$

Now by assuming that $x$ is a function of $v$, Equation (30) is written into the form

$$
\frac{d x}{d v}+\frac{-f_{1}(v)}{f_{2}(v)-v f_{1}(v)} x=\frac{f_{3}(v)}{f_{2}(v)-v f_{1}(v)} x^{k+2} .
$$

Equation (31) is clearly the well known Bernoulli non-linear equation and so by setting $w=x^{-k-1}$, where $w$ and $x$ are all functions of $v$, then Equation (31) turns out as

$$
\frac{d w}{d v}+\frac{(k+1) f_{1}(v)}{f_{2}(v)-v f_{1}(v)} w=\frac{-(k+1) f_{3}(v)}{f_{2}(v)-v f_{1}(v)}
$$

This is clearly the standard non-exact differential equation. Now per case 1 , if $f(x)$ is replaced with

$$
\frac{(k+1) f_{1}(v)}{f_{2}(v)-v f_{1}(v)}=\frac{(k+1) x f_{1}(y / x)}{x f_{2}(y / x)-y f_{1}(y / x)}
$$

then the transformed Equation (32) when written out in its differential form will possess the integrating factor given by

$$
\mu(x, y)=e^{\int \Lambda d(y / x)},
$$


where $\Lambda=\frac{(k+1) x f_{1}(y / x)}{x f_{2}(y / x)-y f_{1}(y / x)}$

\section{Discussion of results}

An erroneous impression usually created in the study of differential equations lies in the result of solving the partial differential equation from which an integrating factor could be extracted as being relatively difficult to handle than the original non-exact equation to which a solution is being sorted. The general rule for solving a differential equation is to identify its type and to map up the method that best solves it. However, many differential equations do not fall under the category of separability, homogeneity, and exactness and so require some integrating factors to reduce them to any of such types that lead to their solutions. In general, there are no known algorithms for finding integrating factors and so it is essential to explore their derivations and not to resign them to only the simple cases involving either the independent or the dependent variable only. An advantage that can be adduced about integrating factors is that it may happen that at least two integrating factors $\mu_{1}, \mu_{2}$ to the particular non-exact differential equation can be easily found from any of the cases outlined, such that their ratio is not a constant, then it will be superfluous and waste of effort to go through the normal procedure in finding the potential function that solves the differential equation. In such instance, the general solution is best written out as an implicit relation in the form $\mu_{1}(x, y)=C \mu_{2}(x, y)$. This can be anticipated in the sense that, a partial differential equation may have more than one possible solution to it, and each of the integrating factors $\mu_{1}, \mu_{2}$ satisfies the established partial differential equations at Equation (2). The constant $C$ has to do with the associated order of the original differential equation.

We adopted the ratio theorem together with specific multipliers to derive some integrating factors and further observed keenly that certain differential forms were equally exploitable in advancing other important integrating factors. Generally integrating factors are not unique, for the single fundamental reason that the partial differential equation from which these integrating factors are developed from do not have unique solutions and so such intrinsic characterization are exhibited by them as well.

It is important to underscore that not, every integrating factor turns out as an exponential function as may be observed for the cases 7,8 and 9 . These cases have been meticulously introduced to give a firm grip on the subject matter. They are developed from the viewpoint of certain peculiarity of their forms and characterization such as the homogeneity of their coefficients and even much more on the instance that some are easily turned into product of exact forms.

Case 9 was more or less developed on a hunch base on the form of the coefficients of the differential pairs with the condition that the determinant of the resulting linear equations should be non-singular. Another fair form of case nine with arbitrary functions whose total derivatives are coincidental to the equation expressed in differential form was also exploited to give a simpler integrating factor for that particular case.

Finally, the case 10 was introduced to show that not every differential equation in differential form may have direct integrating factor related to it. However, with appropriate substitutions, they transform into named differential equation such as the Bernoulli equation which is known to have some integrating factor linked to it when transformed into the standard first-order differential equation.

\section{Conclusion}

In this paper, an exploration is made into the derivation of integrating factors that are dependent on certain linear combinations involving two variables. Such useful functions are not unique in general, and the particular ordinary differential equation that calls for its use may have an infinite number of them associated with the very same differential equation. Thus, with some dexterity and ingenuity, any of such integrating factors could be applied to turn the defining non-exact differential equation into solvable type without recourse to the usual forms they are adopted either in terms of the single independent variable $x$ or that of the dependent variable $y$. We hope that the research on the gallery of integrating factors will spark a renewed interest and also make the subject matter have its rightful place it fully deserves in the study of differential equation.

Author Contributions: All authors contributed equally to the writing of this paper. All authors read and approved the final manuscript.

Conflicts of Interest: "The authors declare no conflict of interest." 


\section{References}

[1] Cheb-Terrab, E. S., \& Roche, A. D. (1999). Integrating factors for second-order ODEs. Journal of Symbolic Computation, 27(5), 501-519.

[2] Ghiasi, E. K., \& Noeiaghdam, S. (2020). Truncating the series expansion for unsteady velocity-dependent Eyring-Powell fluid. Engineering and Applied Science Letter, 3(4), 28-34.

[3] Haq, E. U., Ali, M., \& Khan, A. S. (2020). On the solution of fractional Riccati differential equations with variation of parameters method. Engineering and Applied Science Letter, 3(3), 1-9.

[4] Howell, K. B. (2019). Ordinary Differential Equations: An Introduction to the Fundamentals. CRC Press.

[5] Boyce, W. E., DiPrima, R. C., \& Meade, D. B. (2021). Elementary Differential Equations and Boundary Value Problems. John Wiley \& Sons.

[6] O’Neil, V. P. (2012). Advanced Engineering Mathematics. Cengage Learning, Stamford, USA.

[7] Dass, H. K. (2008). Advanced Engineering Mathematics. S. Chand Publishing.

[8] Beccar-Varela, M. P., Bhuiyan, M. A. M., Mariani, M. C., \& Tweneboah, O. K. (2019). Analytic methods for solving higher order ordinary differential equations. Mathematics, 7(9), 826, https://doi.org/10.3390/math7090826.

[9] Hydon, P. E. (2006). Introduction to symmetry methods in the solution of differential equations that occur in chemistry and chemical biology. International journal of quantum chemistry, 106(1), 266-277.

[10] Hydon, P. E., \& Hydon, P. E. (2000). Symmetry Methods for Differential Equations: A Beginner's Guide (No. 22). Cambridge University Press.

[11] Andam, E. A., Obeng-Denteh, W., Obiri-Apraku, L., Agyei, W., \& Atteh, E. (2016). Algorithm for integrating factor for a non-exact linear first order ordinary differential equation. International Journal of Engineering and Scientific Research, 4(4), 40-51.

[12] Leach, P. G. L., \& Bouquet, S. É. (2002). Symmetries and integrating factors. Journal of Nonlinear Mathematical Physics, 9(sup2), 73-91.

[13] Edwards, C. H., Penney, D. E., \& Calvis, D. (2008). Student Solutions Manual [to Accompany] Elementary Differential Equations with Boundary Value Problems, [And] Elementary Differential Equations. Pearson Education.

[14] Hajja, M. (2013). The integrating factors of an exact differential equation. Mathematics Magazine, 86(3), $220-226$.

[15] Nagy, G. (2020). Ordinary Differential Equations. Mathematics Dept. Michigan State University, East Lansing.

[16] Kuttler, K. (2017). Elementary Differential Equations. CRC Press.

[17] Potter, M. C., Lessing, J. L., \& Aboufadel, E. F. (2019). Wavelets. In Advanced Engineering Mathematics (pp. 670-698). Springer, Cham.

[18] Roberts, C. (2018). Elementary Differential Equations: Applications, Modelsand Computing, CRC Press.

[19] Kreyszig, E. (2008). Advanced Engineering Mathematics. JohnWileyand sons. Inc., New York.

[20] Tahir-Kheli, R. (2019). Ordinary Differential Equations: Mathematical Tools for Physicists. Springer.

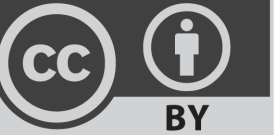

(C) 2021 by the authors; licensee PSRP, Lahore, Pakistan. This article is an open access article distributed under the terms and conditions of the Creative Commons Attribution (CC-BY) license (http://creativecommons.org/licenses/by/4.0/). 